\title{
Cost Effectiveness of Quadrivalent Influenza Vaccines Compared with Trivalent Influenza Vaccines in Young Children and Older Adults in Korea
}

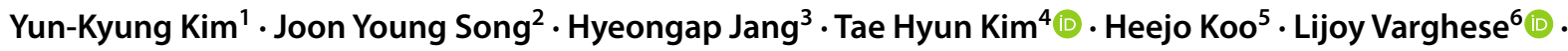 \\ Euna $\operatorname{Han}^{5,7}$ (i)
}

Published online: 25 September 2018

(c) The Author(s) 2018

\begin{abstract}
Introduction Trivalent influenza vaccines (TIVs) are currently reimbursed for subjects aged $\geq 65$ years and children between 6 and 59 months of age under a national immunization program in South Korea. Quadrivalent influenza vaccines (QIVs) are expected to address the potential problem of influenza B-lineage mismatch for TIVs.

Objective The objective of this analysis was to compare the cost effectiveness of QIV versus TIV in children aged 6-59 months and older adults $\geq 65$ years of age in South Korea.

Methods A 1-year static population model was employed to compare the costs and outcomes of a QIV vaccination program compared with TIV in children aged 6-59 months and older adults $\geq 65$ years of age in South Korea. Influenza-related parameters (probabilities, health resource use, and costs) were derived from an analysis of the National Health Insurance System claims database between 2010 and 2013 under a broad and narrow set of International Classification of Diseases, Tenth Revision (ICD-10) codes used to identify influenza. Other inputs were extracted from published literature. Incremental cost-effectiveness ratios (2016 South Korean Won [KRW] per quality-adjusted life-year [QALY] gained) were estimated using a 'limited' societal perspective as per the Korean pharmacoeconomic guidelines. QALYs lost due to premature mortality were discounted at $5 \%$ annually.

Results For both age groups combined, under the narrow definition of influenza, QIV is expected to prevent nearly 16,000 (2923 in children and 13,011 in older adults) medically attended influenza cases, nearly 8000 (672 in children, 7048 in older adults) cases of complications, and over 230 ( 0 in children, 238 in older adults) deaths annually compared with TIV. The impact of using QIV versus TIV in this setting translates into savings of KRW 24 billion (KRW 0.6 billion in children, KRW 23.4 billion in older adults) in annual medical costs, and over 2100 (18 in children, 2084 in older adults) QALYs. Under the broad definition, the corresponding results are over 190,000 (50,697 in children, 140,644 in older adults) influenza cases, over 37,000 (12,623 in children, 24,526 in older adults) complications, 270 deaths ( 0 in children, 270 in older adults), KRW 94.22 billion (KRW 16 billion in children, KRW 78.2 billion in older adults), and over 3500 QALYs saved (316 in children, 3260 in older adults).

Conclusion The use of QIV over TIV was estimated to not be cost effective in children 6-59 months of age, but cost saving in older adults, using the narrow definition of influenza; however, QIV use was cost saving in both age groups using the broad definition. QIV is expected to yield more benefits in older adults $\geq 65$ years of age than in children aged 6-59 months due to higher influenza-related mortality and costs among the older adults. Further analyses considering the indirect effects of influenza vaccination in children are required.
\end{abstract}

Yun-Kyung Kim and Joon Young Song contributed equally as co-first authors.

Electronic supplementary material The online version of this article (https://doi.org/10.1007/s40273-018-0715-5) contains supplementary material, which is available to authorized users.

Extended author information available on the last page of the article 


\section{Key Points for Decision Makers}

Influenza is associated with substantial disease burden in young children and older adults in South Korea.

This study estimated the impact of a switch from trivalent influenza vaccine (TIV) to quadrivalent influenza vaccine (QIV) in young children and older adults, using data from three influenza seasons.

A switch to QIV is expected to prevent significant morbidity and mortality and result in influenza-related healthcare cost savings, especially in older adults.

\section{Introduction}

Influenza is an acute respiratory infection caused by influenza virus. It is estimated that each year $5-10 \%$ of adults and $20-30 \%$ of children worldwide are affected, causing substantial morbidity and mortality [1].

Two virus types, namely influenza $A$ and influenza B, are responsible for recurrent annual epidemics. They are antigenically distinct and do not exhibit cross-immunity, nor do they undergo intertypic genetic reassortment (recombination). Two influenza A subtypes, namely H1N1 and H3N2, have co-circulated with influenza B viruses since 1977, and current trivalent influenza vaccines (TIVs) contain representatives of each A subtype and B virus [2]. The evolution of influenza B viruses is characterized by co-circulation of antigenically and genetically distinct lineages for extended periods of time. Two lineages are defined by phylogenetic relationships of hemagglutinin (HA) genes - the 'B/Victoria lineage' and the 'B/Yamagata lineage' — and viruses of these two lineages have predominated at different times [2].

The World Health Organization's (WHO) Strategic Advisory Group of Experts on Immunization recommends (among others) seasonal influenza vaccination for children aged 6-59 months, older adults, and those with high-risk conditions [1]. Most of the current seasonal influenza vaccines are trivalent, containing strains of two influenza A subtypes (one of each $\mathrm{H} 1 \mathrm{~N} 1$ and $\mathrm{H} 3 \mathrm{~N} 2$ ) and one strain of an influenza B lineage (Victoria or Yamagata), according to recommendations of the WHO. However, the incidence of influenza B can vary dramatically across influenza seasons. In the US, from 2001-2002 through 2010-2011 (excluding the 2009-2010 pandemic season), influenza B was responsible for, on average, $24 \%$ of influenza-positive samples identified during this period (ranging from $<1$ to $44 \%$ ) [3]. Based on European surveillance data, influenza B was, on average, responsible for $23 \%$ of influenza-positive samples (ranging from 1 to $60 \%$ ) [3]. While studies have shown similar clinical manifestations and outcomes in adults with influenza A and B [4, 5], certain studies have observed an increased risk of hospitalization [6] and mortality [7] in children with influenza B compared with those with influenza A, highlighting, at the very least, the importance of equally preventing influenza B.

Furthermore, for trivalent vaccines, antibody responses to the lineage not contained in the vaccine are reduced in adults and diminished in children [8]. In 5 of the 10 influenza seasons in the US between 2001-2002 and 2010-2011, the predominant circulating influenza B lineage was different from that chosen for the vaccine [3]. Thus, influenza vaccination campaigns with TIVs have had limited effectiveness against influenza B epidemics during seasons in which a significant proportion of the disease was caused by the mismatched influenza B strain. To address the problem of influenza B mismatch, quadrivalent influenza vaccines (QIVs) were developed and licensed globally. In addition to strains of the two influenza A subtypes, QIVs contain strains from both type B lineages (Victoria and Yamagata), potentially offering broader protection.

In South Korea, the Korea Centers for Disease Control $\&$ Prevention (KCDC) recommends annual vaccinations for those at high risk. As part of the Korean National Immunization Program (NIP), seasonal influenza vaccines are reimbursed each year during the flu season for adults $\geq 65$ years of age, and more recently (for the 2017-2018 flu season) for children between 6 and 59 months of age [9]. Only TIV is reimbursed in the aforementioned age groups as a part of the NIP for the 2017-2018 flu season; QIV can be reimbursed in the $\geq 65$ years age group on exception (for example, lack of TIV) [10]. The vaccination rate in those aged $\geq 65$ years was $81 \%$ in the year 2015-2016 [11] and $73.3 \%$ for children aged between 1 and 5 years of age [12].

The objective of this study was to determine the cost effectiveness of the QIV influenza vaccine compared with TIV against seasonal influenza in children aged between 6 and 59 months and older adults $\geq 65$ years of age in Korea (Fig. 1).

\section{Methods}

We performed a title/abstract match PubMed review using the search terms 'Korea', 'influenza', 'vaccine' and ('cost effectiveness' or 'cost utility'). No cost effectiveness studies in a South Korean setting for a general population were subsequently identified.

\subsection{Model Structure}

The model employed was a 1-year static decision-tree population model developed in Microsoft Excel (2016; 


\section{Focus on the Patient}

\section{What is the context?}

Influenza is associated with substantial disease burden in young children and older adults. Two distinct influenza virus types; influenza A (common strains: H1N1 and H3N2) and influenza B (two lineages: B/Yamagata and B/Victoria), are responsible for recurrent annual epidemics. Trivalent influenza vaccines (TIVs) contain strains of two influenza A subtypes and one strain of an influenza B lineage. However, the prevailing influenza B lineage for the current season can be different from the lineage present in the vaccine resulting in diminished protection. To address this problem, quadrivalent influenza vaccines (QIVs) were developed.

\section{What is new?}

As part of the Korean National Immunization Program (NIP), TIV is provided each year for older adults and young children. This study estimated the impact of a switch from TIV to QIV in the eligible age-groups using data from three influenza seasons. A switch to QIV is expected to prevent significant morbidity, mortality and result in influenza-related healthcare cost savings.

\section{What is the impact?}

Inclusion of QIV as a part of the Korean NIP against seasonal influenza is expected to address the limitations of TIV. This added protection is significant in older adults where the morbidity and mortality of influenza is highest.

Fig. 1 Focus on the Patient

Microsoft Corporation, Redmond, WA, USA) assessing the cost effectiveness of QIV vaccination strategy against seasonal influenza versus TIV strategy (Fig. 2). Details regarding the model used have been previously published [13] and its validity (structural and conceptual) [14] assessed by an internal panel of cross-functional experts (functions

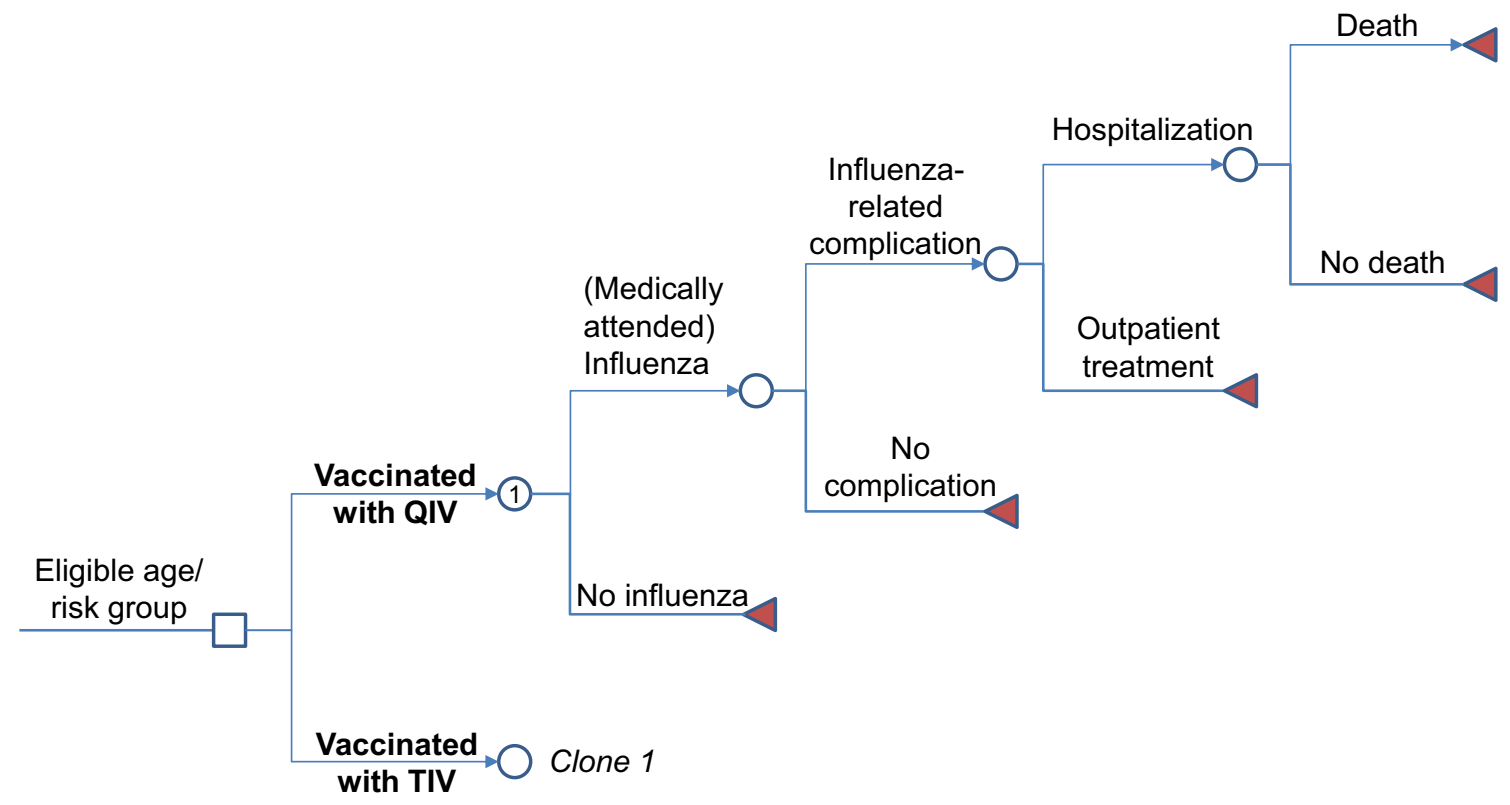

Fig. 2 Decision tree used to compare QIV with TIV. QIV quadrivalent influenza vaccine, $T I V$ trivalent influenza vaccine 
including, but not limited to, medical, epidemiology, and statistics).

The accumulated costs and outcomes over a 1-year period of two age groups (viz 6-59 months and $\geq 65$ years) vaccinated with QIV were compared with those vaccinated with TIV. Since the model only compared the cohorts over a 1-year period, no discounting was applied to either costs or outcomes; quality-adjusted life-years (QALYs) lost due to premature death from an influenza complication were discounted at $5 \%$ in accordance with the Korean pharmacoeconomic guidelines $[15,16]$. In the base-case, the model included both costs reimbursed by the National Health Insurance and out-of-pocket expenses incurred by a patient based on a 'limited' societal perspective as per the Korean pharmacoeconomic guidelines [17]. Thus, direct non-medical costs and indirect costs (for example, productivity losses of the individual or caregiver) were not included in the analysis.

\subsection{Demographics}

The age-specific Korean population [18] estimates for 2016 were obtained from the Korean Statistical Information Service.

\subsection{Epidemiology}

To determine the incidence of seasonal influenza in Korea, a sample cohort of approximately 1 million individuals enrolled in the Korean National Health Insurance Service (NHIS) was used for analysis. The NHIS consists of two major healthcare programs for universal coverage of all residents of Korea-National Health Insurance, and Medical Aid. Approximately 97\% of the population is covered by National Health Insurance, and the remaining 3\% of the population is covered by Medical Aid [19].
For the study, a sample cohort from 2010 to 2013 was used. Three waves were generated: October 2010-April 2011; October 2011-April 2012; and October 2012-April 2013. Based on their age at 15 January 2010, individuals were grouped into the following age groups: $\leq 59$ months and $\geq 65$ years.

Based on an evaluation conducted by the National Evidence-based Healthcare Collaborating Agency (NECA) [20], seasonal influenza was identified by a set of International Classification of Diseases, Tenth Revision (ICD-10) codes mentioned as either a primary or secondary diagnosis in the corresponding individual's claim. Further ICD-10 codes added to the initial list based on the authors' clinical discretion and two definitions, each with its own code set, were arrived at to retrospectively identify influenza from the NHIS (electronic supplementary Table 9). The two definitions were expected to represent broad to narrow definitions for seasonal influenza. Any diagnosis codes with pathogen and without influenza in the description were excluded among the aforementioned ICD-10 codes.

The incidence of influenza for each wave was defined as the proportion of sample persons identified with influenza (ICD-10 codes are presented in electronic supplementary Table 9) among the total number of sample persons in each age group per wave. The incidence rate was then averaged across the three waves. Similarly, the average probability of hospitalization was calculated as the proportion of individuals identified with influenza who used any inpatient services with regard to influenza-related complications (ICD-10 codes are presented in electronic supplementary Table 10) among the total number of sample persons in each age group for each of the three waves (Table 1).
Table 1 Age-specific epidemiological probabilities

\begin{tabular}{|c|c|c|c|c|}
\hline & \multicolumn{2}{|l|}{$6-59$ months } & \multicolumn{2}{|l|}{$\geq 65$ years } \\
\hline & $\begin{array}{l}\text { Narrow defini- } \\
\text { tion }(\%)\end{array}$ & $\begin{array}{l}\text { Broad defini- } \\
\text { tion }(\%)\end{array}$ & $\begin{array}{l}\text { Narrow defini- } \\
\text { tion }(\%)\end{array}$ & $\begin{array}{l}\text { Broad } \\
\text { definition } \\
(\%)\end{array}$ \\
\hline Incidence of influenza & 4.64 & 80.41 & 5.52 & 59.68 \\
\hline Complications following influenza & 22.98 & 24.90 & 54.17 & 17.44 \\
\hline Being a newly diagnosed complication & 99.19 & 99.26 & 86.57 & 83.04 \\
\hline Complication being an exacerbation & 0.81 & 0.74 & 13.43 & 16.96 \\
\hline \multicolumn{5}{|l|}{ Case-fatality rates } \\
\hline Inpatient complications & 0.00 & 0.00 & 6.48 & 2.07 \\
\hline Outpatient complications & 0.00 & 0.00 & 0.72 & 0.24 \\
\hline Non-complicated influenza ${ }^{a}$ & 0.00 & 0.00 & 8.43 & 0.71 \\
\hline
\end{tabular}

Based on the analysis of the National Health Insurance Service database using the International Classification of Diseases codes identified by experts and previous publications [20, 21]

${ }^{\mathrm{a}}$ Assumed to be zero in the base-case analysis 


\subsubsection{Influenza-Related Complications}

Complications associated with a case of influenza were categorized as 'newly diagnosed complications' and 'exacerbation of existing chronic diseases' based on the authors' clinical discretion and a previous analysis on the burden of viral respiratory disease in Korea [21]. The definitions of the categories are:

- Newly diagnosed complications: Hospitalization or outpatient visit with defined disease codes within 4 weeks after an influenza event and having no defined disease codes (inpatient and outpatient) within 1 year before an influenza event.

- Exacerbation of existing chronic conditions: Hospitalization with defined disease codes (main codes) within 4 weeks after an influenza event and having no hospitalization with defined disease codes within 1 year before an influenza event.

Based on the ICD-10 codes used to define the corresponding influenza-related complications for the various age groups (electronic supplementary Table 10), and the corresponding influenza cases for those age groups, the probabilities of developing complications were estimated (Table 1).

\subsubsection{Mortality}

The NHIS provides linked death records for the sample cohort from the Korean National Statistical Office to inform causes of death [22]. Influenza-related mortality was defined as any death occurring within 2 months after influenza diagnosis, and was estimated for the various influenza outcomes (Table 1).

We observed that the mortality ratio for the non-complicated influenza cases in the older adults ( $\geq 65$ years of age) was higher than those with complications resulting in hospitalization. A possible reason for this observation could be that for either of the two earlier-defined complication categories a conservative restriction of no hospitalization for the corresponding
ICD codes for a period of 1 year prior to the current influenza diagnosis was applied. In particular, for older individuals with chronic medical conditions with frequent hospitalizations, this restriction might not take into account 'true' influenza-related exacerbations (which could potentially be identified through a medical chart review). Thus, individuals with cases classified as non-complicated based on the complication criteria listed earlier might have been misclassified and this might explain the reason for the high mortality rates. Hence, for the basecase analysis, we assumed no mortality for non-complicated influenza across all ages.

\subsubsection{Influenza Circulation}

The proportion of influenza B strains among the overall influenza-positive cases identified each year were obtained from the Korean Influenza Surveillance Scheme reports [23]. The subsequent breakdown of the influenza B lineages for each of those years was obtained from a study that looked at the epidemiology of influenza B disease in various Asia-Pacific countries [24]. The percentage of influenza B mismatch for TIV is calculated as $1-$ (percentage of the B-lineage included in TIV) that was observed circulating in that corresponding year. The 2009-2010 season was excluded due to the predominantly pandemic influenza strain circulation in that season.

The B-lineage distribution was not reported for two seasons, i.e. 2008-2009 and 2010-2011. To impute the missing values, the average of the other four seasons was assumed. Finally, simple averages of the TIV influenza B mismatch and the proportion of influenza $B$ circulating over six influenza seasons were estimated (Table 2). If weighted for the proportion of B circulation across the six seasons, the weighted average of B-lineage mismatch was $73.5 \%$; however, the simple average value was used to model a more conservative estimate.

\subsection{Costs}

We extracted cost data from insurance claims for the aforementioned three waves (October 2010-April 2011; October 2011-April 2012; and October 2012-April 2013) and
Table 2 Influenza B distribution and vaccine B-lineage mismatch in Korea

\begin{tabular}{llrlcr}
\hline Year & Vaccine lineage & Type B (\%) & \multicolumn{2}{c}{ Circulating lineage (\%) } & \multirow{2}{*}{ Mismatch (\%) } \\
\cline { 4 - 5 } & & & 0.0 & 100.0 & 100.0 \\
\hline $2007-2008$ & Victoria & 64.1 & $55.9^{\mathrm{a}}$ & $44.1^{\mathrm{a}}$ & 55.9 \\
$2008-2009$ & Yamagata & 1.2 & $55.9^{\mathrm{a}}$ & $44.1^{\mathrm{a}}$ & 44.1 \\
$2010-2011$ & Victoria & 0.9 & 73.0 & 27.0 & 27.0 \\
$2011-2012$ & Victoria & 48.5 & 64.6 & 35.4 & 64.6 \\
$2012-2013$ & Yamagata & 5.6 & 86.0 & 14.0 & 86.0 \\
$2013-2014$ & Yamagata & 53.0 & & & 62.9 \\
Average & & 28.9 & & & \\
\hline
\end{tabular}

${ }^{\mathrm{a}}$ Imputed values are based on the B-lineage average for other seasons 
adjusted it to 2016 South Korean Won (KRW) using the Korean gross domestic product (GDP) deflators [25].

For the cost of inpatient stay, we identified any claims for patients who used inpatient services with regard to influenza-related complications (ICD-10 codes are presented in electronic supplementary Table 10) and summed the cost per patient in order to obtain the mean cost of the inpatient stay per person. The cost of outpatient treatment was similarly calculated as the cost of inpatient stay, using the cost of the outpatient service instead of cost per person.

For each category of complication, the average cost of inpatient stay included hospitalization and related medication costs. The average length of stay (ALoS; in days) was estimated based on the length of the inpatient stay for each complication per influenza season (Table 3).

In the case of a complication that required only outpatient treatment, the average cost was estimated based on the corresponding hospital consultation and medication costs. No outpatient visits were assumed for complications that were exacerbations of existing chronic conditions.

For non-complicated influenza cases (defined as all influenza cases identified without the codes listed in electronic supplementary Table 10), the corresponding costs for outpatient and inpatient services were included.

\subsubsection{Vaccine and Antiviral Costs}

Vaccine costs were assumed to be KRW 7510 for TIV (based on the 2016 NIP procurement price) [26] and KRW 11,265 for QIV (assuming a 50\% premium due to the inclusion of an additional B lineage). No administration costs were considered as the same dosage and coverage was assumed for both vaccines.

Antivirals (neuraminidase inhibitors [NIs]) are usually prescribed for an influenza event; however, the NHIS only reimburses a portion of the costs based on the age and risk category of the individual [27, 28]. The antiviral prescriptions and average reimbursed cost per prescription were extracted from the NHIS sample cohort. The out-of-pocket costs for antivirals were included in the analysis. A price of KRW 30,000 was assumed for a 5-day course of antivirals (based on the list price in the NHIS for oseltamivir $75 \mathrm{mg}$, 5-day course; i.e. 10 tablets per course-KRW 2170 per tablet—and a pharmacy preparation cost of KRW 6070; the total cost [KRW 27,770] was rounded up to KRW 30,000)
Table 3 Age-specific direct medical costs for influenzarelated outcomes

\begin{tabular}{|c|c|c|c|c|}
\hline & \multicolumn{2}{|c|}{ Narrow definition } & \multicolumn{2}{|c|}{ Broad definition } \\
\hline & $6-59$ months & $\geq 65$ years & $6-59$ months & $\geq 65$ years \\
\hline \multicolumn{5}{|l|}{ Newly diagnosed complications } \\
\hline Probability of hospitalization (\%) & 28.18 & 37.83 & 25.41 & 36.17 \\
\hline Mean cost of inpatient stay (KRW) & 729,875 & $4,298,958$ & 760,918 & $4,303,776$ \\
\hline ALoS (days) & 6.95 & 19.80 & 7.20 & 19.46 \\
\hline Mean cost of outpatient treatment (KRW) & 63,633 & 123,083 & 66,547 & 149,819 \\
\hline \multicolumn{5}{|l|}{ Exacerbation of existing chronic conditions } \\
\hline Probability of hospitalization (\%) & 100 & 100 & 100 & 100 \\
\hline Mean cost of inpatient stay (KRW) & $2,861,959$ & $4,192,447$ & $4,664,615$ & $3,201,406$ \\
\hline ALoS (days) & 7.79 & 31.45 & 10.62 & 22.39 \\
\hline Mean cost of outpatient treatment (KRW) & - & - & - & - \\
\hline \multicolumn{5}{|l|}{ Non-complicated influenza cases } \\
\hline Mean cost of treatment (KRW) & 158,127 & $1,450,271$ & 290,571 & 232,767 \\
\hline
\end{tabular}

$A L O S$ average length of stay, KRW South Korean Won

Table 4 Estimated out-of-pocket costs for antivirals

\begin{tabular}{llllll}
\hline & $6-59$ months & & & $\geq 65$ years \\
& Narrow definition & Broad definition & & Narrow definition & Broad definition \\
\hline OOP NI costs for non-complicated cases (KRW) & 22,276 & 29,545 & 29,248 & 29,916 \\
OOP NI costs for complicated cases (KRW) & 24,868 & 29,720 & & 29,766 & 29,866 \\
Non-complicated influenza cases (\%) & 77.02 & 75.10 & 45.83 & 82.56 \\
Complicated influenza cases (\%) & 22.98 & 24.90 & 54.17 & 17.44 \\
Overall OOP NI costs (KRW) & 22,872 & 29,589 & 29,529 & 29,908 \\
\hline
\end{tabular}

$N I$ neuraminidase inhibitor, $O O P$ out-of-pocket, $K R W$ South Korean Won 
[29]. All individuals seeking treatment for influenza were assumed to receive a 5-day course of antivirals [27, 30]. Based on the reimbursed costs obtained from the NHIS analysis, as well as the assumed cost of the antiviral prescription, the out-of-pocket costs were estimated for each age group (see the 'Estimation of Out-of-Pocket Antiviral Costs' section in the electronic supplementary material) (Table 4).

\subsection{Vaccine Efficacy (VE)}

The model incorporates vaccine efficacy (VE) against influenza separately as efficacy against Influenza A and Influenza B. For TIV, the efficacy against the latter is broken down as efficacy against a B-lineage match and mismatch scenario.

$$
\begin{aligned}
& \text { Vaccine efficacy, } \mathrm{VE}_{\mathrm{TIV}, \text { overall }} \\
& \begin{aligned}
= & \mathrm{VE}_{A} \cdot p_{A}+\left(\mathrm{VE}_{\mathrm{B}, \text { mismatch }} \cdot p_{\mathrm{B}-\text { mismatch }}\right. \\
& \left.+\mathrm{VE}_{\mathrm{B}, \text { match }} \cdot\left(1-p_{\mathrm{B} \text {-mismatch }}\right)\right) \cdot\left(1-p_{A}\right)
\end{aligned}
\end{aligned}
$$

where $p_{\mathrm{A}}=\%$ influenza A identified among circulating influenza-positive samples identified, $\mathrm{VE}_{\mathrm{A}}=$ vaccine efficacy against Influenza $\mathrm{A}, p_{\mathrm{B} \text {-mismatch }}=$ percentage of influenza $B$ identified among circulating influenza $B$ that does not match the $\mathrm{B}$-lineage included in the vaccine, and $\mathrm{VE}_{\mathrm{B} \text {-mismatch }}$ = vaccine efficacy against Influenza B for the B-lineage not included in the vaccine.

\subsubsection{VE Against Influenza A}

The reported VEs against influenza can vary depending on the study design, outcomes measured, population studied, and the season in which the flu vaccine was studied. To obtain an unbiased estimate of VE for the various age groups, meta-analyses of randomized controlled trials for TIV were used (see the 'Estimation of Vaccine Efficacies' section in the electronic supplementary material) [31-33]. TIV VE against influenza $A$ was assumed to be the same as that of the overall VE reported against influenza as neither of the meta-analyses in children or older adults assessed efficacy against influenza A separately. Both TIV and QIV contain the same WHO-recommended influenza A strains each year. Subsequently, VE against influenza A for both vaccines in both age groups was assumed to be the same. A recently concluded QIV efficacy trial in children aged 6-35 months reported that QIV efficacy against seasonal influenza of any severity was $50 \%$ [34]. Nonetheless, we assumed the same VE for QIV as that estimated for TIV for children between 6 and 59 months of age.

\subsubsection{VE Against Influenza B}

VE for TIV against influenza B in adults (18-64 years of age) against vaccine-matched (77\%) and mismatched $(52 \%)$ influenza B lineages was estimated from a meta-analysis [8].

Since no estimate of TIV efficacy against matched and mismatched B lineages were available for children and older adults, the relative overall efficacy proportion of TIV against influenza relative to adults (as described in Table 5) was applied to the efficacies against reported by Tricco et al. [8]. Please refer to the 'Estimation of Vaccine Efficacies' section in the electronic supplementary material for more details.

Due to the static nature of the model, no herd effect was incorporated in the model.

VE for QIV against Influenza B is assumed to be the same as that of TIV against a matched B-lineage scenario [35].
Table 5 Age-specific vaccine efficacy assumptions for

\begin{tabular}{|c|c|c|c|c|c|c|}
\hline \multirow[t]{3}{*}{ Age group } & \multicolumn{2}{|c|}{$\begin{array}{l}\text { Vaccine efficacy } \\
\text { against influenza A }\end{array}$} & \multicolumn{4}{|c|}{ Vaccine efficacy against influenza B } \\
\hline & \multirow[t]{2}{*}{ TIV } & \multirow[t]{2}{*}{ QIV } & \multicolumn{2}{|l|}{ TIV } & \multicolumn{2}{|l|}{ QIV } \\
\hline & & & Matched & Mismatched & Matched & Mismatched \\
\hline $6-59$ months & $0.47^{\mathrm{a}}$ & $0.47^{\mathrm{a}}$ & $0.58^{\mathrm{c}}$ & $0.39^{c}$ & $0.58^{\mathrm{d}}$ & NA \\
\hline$\geq 65$ years & $0.58^{\mathrm{b}}$ & $0.58^{\mathrm{b}}$ & $0.72^{\mathrm{c}}$ & $0.49^{\mathrm{c}}$ & $0.72^{\mathrm{d}}$ & NA \\
\hline
\end{tabular}
influenza A and B with TIV or QIV
$N A$ not applicable, $Q I V$ quadrivalent influenza vaccine, $T I V$ trivalent influenza vaccine, $V E$ vaccine efficacy ${ }^{\text {a} P o o l e d ~ e s t i m a t e ~(u s i n g ~ a ~ r a n d o m-e f f e c t s ~ m o d e l) ~ o f ~ o u t c o m e s ~ i n ~ c h i l d r e n ~}<6$ years of age, as reported by Jefferson et al. [33]

${ }^{b}$ Pooled estimate of randomized controlled trials of parenteral vaccine versus placebo in the review by Jefferson et al. [32]

${ }^{\mathrm{c}}$ Proportional estimate obtained by multiplying the ratio of VE (in adults) against influenza B in matched/ mismatched scenarios (as reported by Tricco et al. [8]) and the VE (in adults) against influenza (as reported by Demicheli et al. [31]; 63\%) with the VE assumed against influenza A for the particular age group (see footnote ' $a$ ' or ' $b$ ')

${ }^{\mathrm{d}}$ Assumed to be the same as the matched B-lineage scenario for TIV [35] 


\subsubsection{Vaccine Coverage}

Yang and Cho reported influenza vaccine coverage of $81.2 \%$ and $83.1 \%$ in the $65-74$ years and $\geq 75$ years age groups for the 2011-2012 influenza season, based on the Korean Community Health Survey [36]. Adjusting for population sizes, the average coverage in the $\geq 65$ years age group was calculated to be $82 \%$. A study that assessed the Korea National Health and Nutrition Examination Survey estimated that the influenza vaccine coverage in children $<5$ years of age was $78.9 \%$ in 2014 [37]. Since vaccination was free in this age group as a part of the universal mass vaccination program from the 2017-2018 season, the same coverage in older adults was assumed for the 6-59 months age group.

\subsection{Utilities}

A disutility of 0.44 was applied for any influenza episode irrespective of whether the episode was complicated or uncomplicated, or if it also required hospitalization or resulted in an outpatient visit $[38,39]$. To estimate the QALY loss for hospitalized complications, the ALoS estimated from the NHIS data sample was used. The overall QALY loss for hospitalized complicated influenza cases was calculated based on the ALoS weighted for the ratio of newly diagnosed complications to exacerbations of chronic conditions for each age group (Table 6). Complicated influenza treated in an outpatient setting, as well as non-complicated influenza, were assumed to last 5 days irrespective of the age of the individual.

To estimate the discounted QALYs lost due to premature influenza-related death, the age-specific Korean healthadjusted life expectancies (HALE) for 2011 were used [40]. Because HALE was reported for 5-year age groups, a quadratic curve was fitted to the reported data to obtain an estimate for HALE for single-year age groups. Next, for each single-year age group, the present value of the HALE was estimated using the annuity method discounted at various rates, i.e. 0,3 , and $5 \%$. Finally, to estimate the average discounted HALE for each of the aforementioned age groups, a population-weighted approach was used (Table 6).

\subsection{Sensitivity Analysis}

A major parameter that was expected to determine the results was the extent of the B-lineage mismatch. To assess the impact of the variation in B-mismatch on the cost-effectiveness outcomes, two scenarios were tested:

- Low mismatch: B-mismatch assumed to be $0 \%$.

- High mismatch: B-mismatch assumed to be $100 \%$.

A univariate analysis was performed by varying each parameter over the $95 \%$ confidence intervals using the
Table 6 Age-specific QALY loss estimates for influenzarelated outcomes

\begin{tabular}{|c|c|c|c|c|}
\hline & \multicolumn{2}{|c|}{ 6-59 months } & \multicolumn{2}{|c|}{$\geq 65$ years } \\
\hline & $\begin{array}{c}\text { Narrow } \\
\text { definition }\end{array}$ & $\begin{array}{c}\text { Broad } \\
\text { definition }\end{array}$ & $\begin{array}{c}\text { Narrow } \\
\text { definition }\end{array}$ & $\begin{array}{c}\text { Broad } \\
\text { definition }\end{array}$ \\
\hline \multicolumn{5}{|l|}{ HOSPITALISED CASES } \\
\hline $\begin{array}{l}\text { ALoS - Newly diagnosed } \\
\text { complications }\end{array}$ & 6.95 & 7.2 & 19.8 & 19.46 \\
\hline $\begin{array}{l}\text { ALoS - Exacerbations of chronic } \\
\text { conditions }\end{array}$ & 7.79 & 10.62 & 31.45 & 22.39 \\
\hline QALY loss & 0.0084 & 0.0088 & 0.0281 & 0.0248 \\
\hline \multicolumn{5}{|l|}{ OUTPATIENT CASES } \\
\hline $\begin{array}{l}\text { Duration - Newly diagnosed } \\
\text { complications }\end{array}$ & \multicolumn{4}{|c|}{5} \\
\hline QALY loss & \multicolumn{4}{|c|}{0.006} \\
\hline Duration - Non-complicated influenza & \multicolumn{4}{|c|}{5} \\
\hline QALY loss & \multicolumn{4}{|c|}{0.006} \\
\hline \multicolumn{5}{|l|}{ Population weighted average HALE (years) } \\
\hline $0 \%$ Annual discount rates & \multicolumn{2}{|c|}{69.8} & \multicolumn{2}{|c|}{10.9} \\
\hline $3 \%$ Annual discount rates & \multicolumn{2}{|c|}{29.1} & \multicolumn{2}{|c|}{9.1} \\
\hline $5 \%$ Annual discount rates & \multicolumn{2}{|c|}{19.3} & \multicolumn{2}{|c|}{8.1} \\
\hline
\end{tabular}

$A L O S$ average length of stay, $H A L E$ health-adjusted life expectancies, $Q A L Y$ quality-adjusted life-year 
underlying probability distributions listed in electronic supplementary Table 15 . A probabilistic sensitivity analysis was performed using a Monte-Carlo simulation of over 2000 iterations for either of the influenza case definitions. A $99 \%$ correlation was assumed between vaccine efficacies for TIV against vaccine $B$ strain match and mismatch scenarios. The rationale for the assumption is that a higher efficacy against influenza $B$ in a match scenario should facilitate higher protection in the $\mathrm{B}$ mismatch scenario and vice versa. The approach used to force correlations between these parameters has been previously published [41].

\section{Results}

\subsection{Base-Case Scenario}

\subsubsection{Narrow Case Definition}

In the base-case scenario for children aged 6-59 months, the use of QIV over TIV is expected to result in preventing nearly 3000 cases of influenza and approximately 670 influenza-related complications annually throughout South Korea. The prevented cases result in over KRW 606 million in healthcare costs (defined as the sum of hospitalization, outpatient, treatment of non-complicated influenza, and antiviral costs) and 18 QALYs saved each year. However, the incremental cost-effectiveness ratio (ICER) in this age group is approximately KRW 345 million per QALY gained, compared with a threshold of 1 GDP per capita of KRW $32,038,000$ for the year 2016 [42].

In older adults $\geq 65$ years of age, the use of QIV over TIV is expected to result in preventing nearly 13,000 cases of influenza and approximately 7000 influenza-related complications. The prevented cases result in over KRW 23 billion in healthcare costs and over 2000 QALYs saved each year. The switch to QIV is expected to be cost saving, with an incremental saving of KRW 2.6 billion each year. The complete incremental outcomes are presented in Table 7.

\subsubsection{Broad Case Definition}

Using the broader definition, in the base-case scenario for children $\leq 59$ months of age, the use of QIV over TIV is expected to result in preventing nearly 51,000 cases of influenza and approximately 13,000 influenza-related complications annually. The prevented cases result in over KRW 16 billion in healthcare costs and over 300 QALYs saved each

Table 7 Base-case results for the narrow and broad case definitions

\begin{tabular}{|c|c|c|c|c|c|c|c|c|}
\hline & \multicolumn{4}{|c|}{$6-59$ months } & \multicolumn{4}{|l|}{$\geq 65$ years } \\
\hline & \multicolumn{2}{|c|}{ Narrow definition } & \multicolumn{2}{|c|}{ Broad definition } & \multicolumn{2}{|c|}{ Narrow definition } & \multicolumn{2}{|c|}{ Broad definition } \\
\hline & TIV & QIV & TIV & QIV & TIV & QIV & TIV & QIV \\
\hline Vaccinated & $1,830,328$ & $1,830,328$ & $1,830,328$ & $1,830,328$ & $5,545,530$ & $5,545,530$ & $5,545,530$ & $5,545,530$ \\
\hline Influenza cases & 63,734 & 60,811 & $1,105,485$ & $1,054,788$ & 196,400 & 183,389 & $2,122,925$ & $1,982,281$ \\
\hline Non-complicated cases & 49,086 & 46,835 & 830,251 & 792,176 & 90,011 & 84,048 & $1,752,725$ & $1,636,607$ \\
\hline Influenza complications & 14,648 & 13,976 & 275,234 & 262,611 & 106,389 & 99,341 & 370,200 & 345,674 \\
\hline Hospitalizations & 4213 & 4020 & 71,468 & 68,190 & 49,133 & 45,878 & 173,991 & 162,464 \\
\hline Outpatient & 10,435 & 9957 & 203,766 & 194,421 & 57,256 & 53,463 & 196,209 & 183,211 \\
\hline Influenza deaths & 0 & 0 & 0 & 0 & 3595 & 3357 & 4075 & 3805 \\
\hline Total costs (KRW, millions) & 26,956 & 33,223 & 363,586 & 354,416 & 394,733 & 392,164 & $1,222,115$ & $1,164,733$ \\
\hline Hospitalization & 3327 & 3174 & 62,324 & 59,466 & 209,698 & 195,806 & 679,603 & 634,580 \\
\hline Outpatient & 664 & 634 & 13,560 & 12,938 & 7047 & 6580 & 29,396 & 27,448 \\
\hline No complications & 7762 & 7406 & 241,247 & 230,183 & 130,541 & 121,893 & 407,977 & 380,949 \\
\hline NI costs & 1458 & 1391 & 32,710 & 31,210 & 5799 & 5415 & 63,492 & 59,285 \\
\hline Vaccination costs ${ }^{\mathrm{a}}$ & 13,746 & 20,619 & 13,746 & 20,619 & 41,647 & 62,470 & 41,647 & 62,470 \\
\hline QALYs lost (discounted at 5\%) & 396 & 378 & 6887 & 6571 & 31,466 & 29,382 & 49,202 & 45,942 \\
\hline Incremental costs (KRW, millions) & & 6267 & & -9171 & & -2568 & & $-57,382$ \\
\hline Incremental QALYs & & 18 & & 316 & & 2085 & & 3260 \\
\hline ICER (million KRW/QALY gained) & & 345.4 & & Cost saving & & Cost saving & & Cost saving \\
\hline
\end{tabular}

All costs are reported in millions of KRW

$I C E R$ incremental cost-effectiveness ratio, $K R W$ South Korean Won, $N I$ neuraminidase inhibitors, $Q A L Y$ s quality-adjusted life-years, $Q I V$ quadrivalent influenza vaccine, $T I V$ trivalent influenza vaccine

${ }^{a}$ Does not include administration costs 


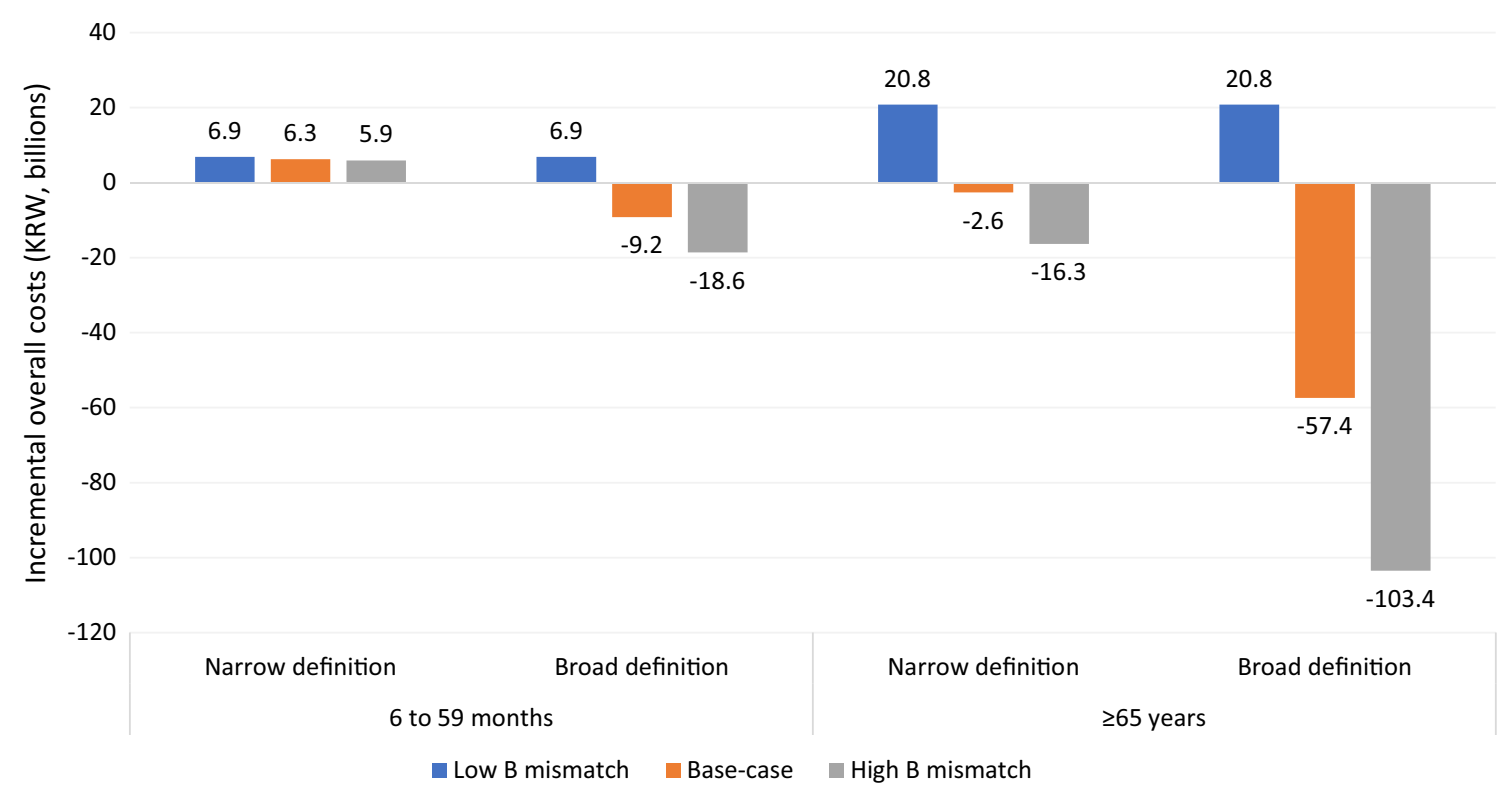

Fig. 3 Impact of B-lineage mismatch on incremental costs between TIV and QIV (narrow and broad definition). KRW South Korean Won, Low B Mismatch B-mismatch assumed to be $0 \%$, High B Mismatch B-mismatch assumed to be $100 \%$

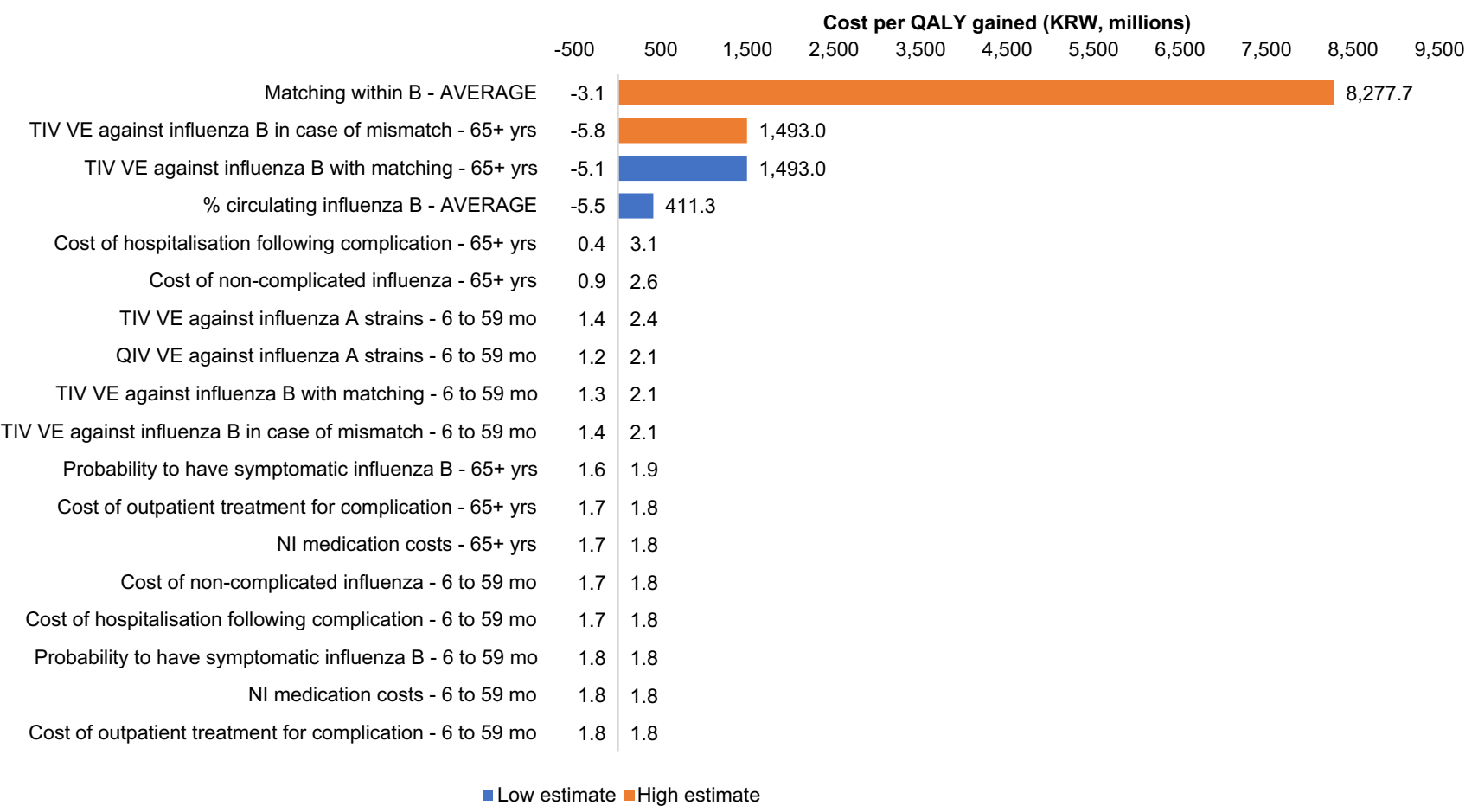

Fig. 4 One-way sensitivity analyses results for the narrow definition for ICER. ICER incremental cost-effectiveness ratio, $K R W$ South Korean Won, mo months, NI neuraminidase inhibitor or antiviral,

year. The switch from TIV to QIV is expected to be cost saving in this age group (incremental annual savings of KRW 9.2 billion).
$Q A L Y$ quality-adjusted life-year, $Q I V$ quadrivalent influenza vaccine, $T I V$ trivalent influenza vaccine, $V E$ vaccine efficacy, $y r s$ years

In older adults aged $\geq 65$ years, the use of QIV over TIV is expected to result in preventing nearly 140,000 cases of influenza and approximately 25,000 influenza-related 


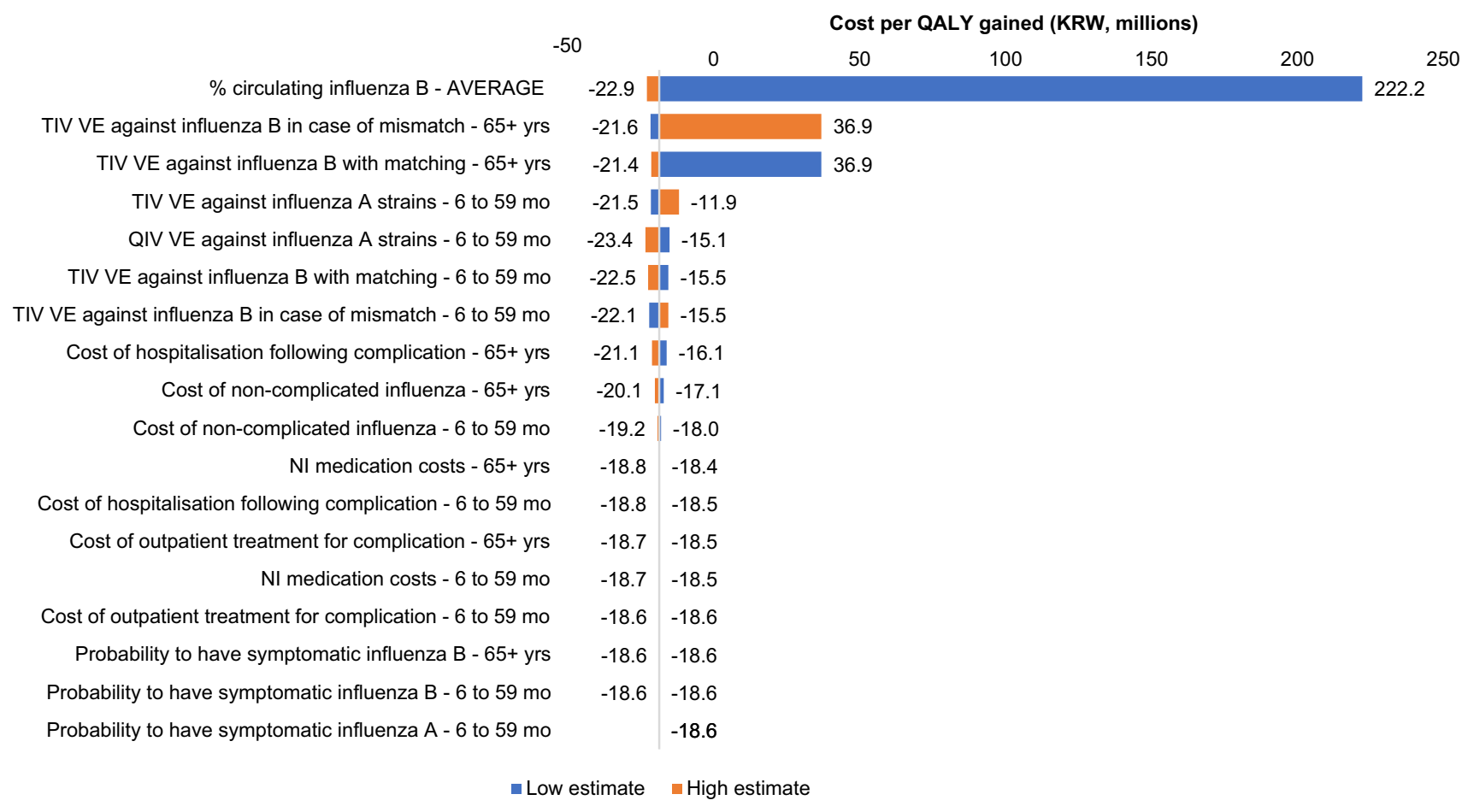

Fig. 5 One-way sensitivity analyses results for the broad definition for ICER. ICER incremental cost-effectiveness ratio, $K R W$ South Korean Won, mo months, NI neuraminidase inhibitor or antiviral,

complications annually. The prevented cases result in over KRW 78 billion in healthcare costs and over 3200 QALYs saved each year. The switch to QIV is expected to be cost saving, with an incremental saving of KRW 57 billion each year. The complete incremental outcomes are presented in Table 7.

\subsection{Variations in B-Mismatch}

In the base-case, the average B-mismatch over the six influenza seasons was used to parameterize the model.

The incremental costs of the switch from TIV to QIV are highly sensitive to variations in B strain mismatch, especially for the $\geq 65$ years age group, as can be seen in Fig. 3 and electronic supplementary Table 16.

\subsection{One-Way Sensitivity Analysis}

The results of the one-way sensitivity analysis (in terms of ICERs) are presented in Figs. 4 and 5. The parameters that have the greatest impact on the incremental costs are the relative efficacies of QIV and TIV against influenza A, the VE against influenza B for TIV in the mismatch scenario, and the proportion of influenza B circulating in the season.
$Q A L Y$ quality-adjusted life-year, $Q I V$ quadrivalent influenza vaccine, $T I V$ trivalent influenza vaccine, $V E$ vaccine efficacy, $y r s$ years

\subsection{Probabilistic Sensitivity Analysis}

The results of the simulations for both definitions of influenza are presented in Fig. 6. As seen in Fig. 7, under the broad definition, $93.9 \%$ and $96.5 \%$ of simulations fell below the 1 GDP per capita willingness to pay (WTP) threshold for children 6-59 months of age and older adults aged $\geq 65$ years, respectively. Under the narrow definition, $92.4 \%$ of simulations were under the WTP threshold for older adults, while none of the simulations in children fell under the threshold.

\section{Discussion}

This study assessed the clinical and economic impact of switching from the currently reimbursed TIV to QIV for two at-risk age groups-children 6-59 months of age and older adults aged $\geq 65$ years. The burden of influenza over three influenza seasons (2010-2013) was estimated using a national sample cohort of the Korean NHIS using two different influenza definitions-a narrow definition and a broader definition.

In the base-case scenario using the narrow case definition, the switch to QIV from TIV is estimated to be cost saving for the population aged $\geq 65$ years, and not cost effective in 
Fig. 6 Cost-effectiveness plane. $G D P$ gross domestic product, $K R W$ South Korean Won, $Q A L Y$ quality-adjusted life-year, $Q I V$ quadrivalent influenza vaccine, $T I V$ trivalent influenza vaccine
Fig. 7 Cost-effectiveness acceptability curve. $K R W$ South Korean Won, $Q A L Y$ qualityadjusted life-year, WTP willingness to pay
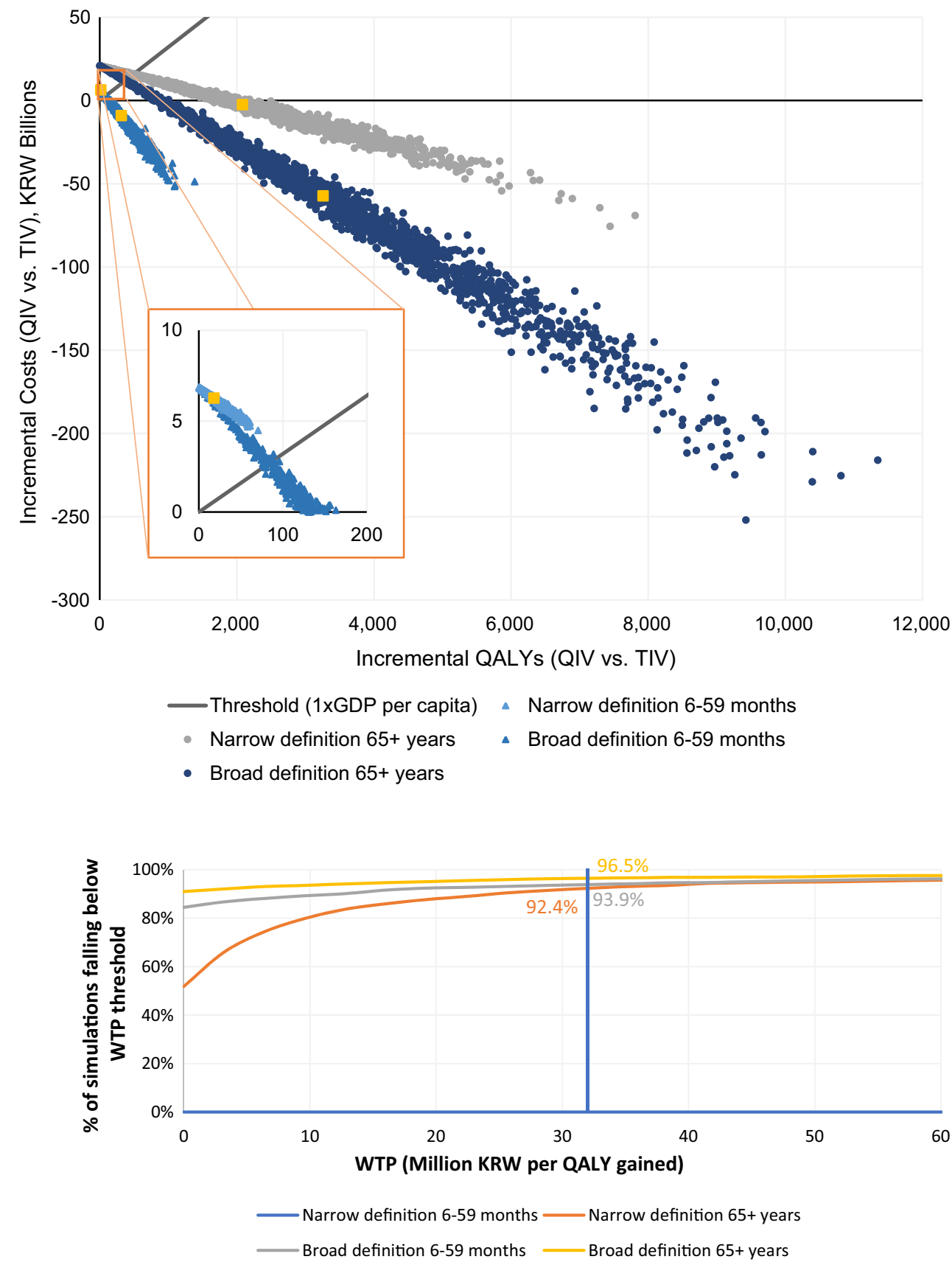

children aged $\leq 59$ months, based on a threshold of 1 GDP per capita for South Korea. However, the switch to QIV was estimated to be cost saving for both age groups using the broad definition.

The contrasting results for children and older adults using the narrow influenza definition despite similar levels of incidence could potentially be explained by four factors:

1. Lower probability of developing complications in children (estimated to be approximately $50 \%$ lower in children).
2. Lower costs of hospitalization (approximately $83 \%$ lower), outpatient treatment (approximately 50\% lower) for newly diagnosed complications and non-complicated influenza (approximately 90\% lower).

3. Lower QALY losses in newly diagnosed complications due to the lower ALoS in children (approximately 65\% lower).

4. No mortality was assumed for children (compared with approximately $2 \%$ overall in the older adults). A $5 \%$ mortality in the case of a hospitalized complication in children would result in an ICER of KRW 30,734,041 
per QALY gained (electronic supplementary Fig. 9), which is almost similar to the WTP threshold of 1 GDP per capita.

Similar discrepancies in resource use with regard to hospitalization were also reported in the previous literature. For example, Thommes et al. reported a 1.39- to 1.66-fold higher hospitalization cost among adults aged 60 years or older compared with children $<8$ years of age in Canada, and a 4.33-fold higher hospitalization cost for those aged 65 years or older in the UK compared with children $<5$ years of age [39]. Similarly, a public health assessment of QIV use over TIV in Latin America observed that the costs of hospitalization in Brazil in those over 60 years of age was 1.34-fold higher than children $<5$ years of age (the rate of hospitalization was also 2.46 -fold higher in older adults compared with children) [43].

As expected, and as evident from the one-way sensitivity analyses, the key differentiator between QIV and TIV, i.e. the efficacy against influenza $B$, was seen to drive the results of the analysis. The proportion of influenza B circulating magnifies the incremental costs and QALYs observed. At the same time, the reported proportion of B circulating was seen to vary significantly, with three of the six seasons seeing an average of $3 \%$ of B circulation and the remaining three an average of $55 \%$.

Despite the sensitivity of the results to the variance in the input parameters, more than $90 \%$ of the simulations for both age groups, using either the broad or narrow definition of influenza, were observed to fall below the WTP threshold of 1 GDP per capita (except for children using the narrow definition). This demonstrates the value of a switch from TIV to QIV, especially in the case of the older adult age group. It has to be noted that, globally, there are no explicit guidelines for an ICER threshold for an intervention to be considered as cost effective [44, 45]. Rather, threshold values for past imbursement decisions are often referred to as experiencebased thresholds for ICER [46, 47]. The pharmacoeconomic guidelines for South Korea also do not suggest an explicit threshold for ICER, but recommend $1 \times$ GDP per capita as the baseline for the threshold, and call for a flexible assessment, taking into consideration disease severity, societal burden, impact on quality of life, and innovativeness of the drug to be assessed [48, 49].

There are limitations to this analysis, with the primary limitation being the accuracy of estimating the incidence of influenza from a database of insurance claims using ICD10 codes. For example, pneumonia cases diagnosed as only a bacterial infection (following a non-medically attended influenza infection) may be missed if influenza was not diagnosed or suspected. To account for this uncertainty, two influenza definitions were used to estimate the annual incidence for all age groups. The broad influenza-like illness definition gave more favorable results, but QIV was estimated to be cost saving in the older adult age group, even when using the narrow definition.

Another limitation was that the herd impacts of influenza vaccination were not considered in the model. The preferred approach to modeling the economic impact of vaccines against infectious diseases (particularly influenza) is the use of dynamic models as they incorporate the indirect effects of the vaccination. Dynamic modeling approaches in France [50], the UK [51], and Germany [52] have shown that extending influenza vaccination to younger age groups (albeit using the live-attenuated influenza vaccine) would result in a significant reduction in morbidity and mortality in all other age groups. In France, a 50\% coverage of liveattenuated influenza vaccine in children 2-17 years of age was expected to result in a further $28 \%$ decrease in cases in those over 18 years of age [50]. Similarly, in a German setting, $60 \%$ of the additional hospitalizations prevented from live-attenuated influenza vaccine use (at 50\% coverage) in the same age group (2-17 years) were estimated to be from those over 18 years of age [52]. Static population models do not allow for the inclusion of herd impact, but, owing to the simplicity of local data requirements, they may be a useful approach where granular local data are limited [53]. However, an exploratory analysis subsequently conducted by varying the indirect effects in the population over 5 years of age by vaccinating the 6-59 months age group alone showed that a $4.5 \%$ indirect effect in preventing influenza in the nonvaccinated age group would result in QIV being cost effective relative to TIV in children (electronic supplementary Fig. 10). Nonetheless, because of the incremental nature of the base-case analysis, and the fact that QIV was expected to be at least as effective as TIV against influenza, the lack of inclusion of indirect effects should result in a conservative result for QIV [53, 54].

The productivity gains from a switch to QIV were not included in this analysis. Even though the two age groups (children $\leq 59$ months and older adults $\geq 65$ years) have little or no employment, an episode of influenza is expected to result in some productivity loss for a parent (in the case of a child) or a caregiver. The inclusion of productivity losses should result in an increased estimate of the value of QIV over TIV.

Lastly, improvements in the quality of care and reduced strain on the healthcare system could be expected from a reduction of healthcare resources sought during the peak winter periods of an influenza season; these indirect effects were not included in the analysis.

Despite these limitations, the results of the current analysis (i.e. a switch from TIV to QIV is expected to be cost effective) are concordant with the global body of evidence $[39,51,55-61]$. 


\section{Conclusions}

QIV use is expected to lead to increased public health improvements over TIV use in the 6-59 months and $\geq 65$ years age groups using both a narrow and broad definition of influenza. In children aged 6-59 months, a switch to QIV is expected to be cost saving using the broad definition, but not cost effective using the narrow definition due to the lack of mortality assumed in the age group and the non-inclusion of indirect protection. However, QIV was estimated to be cost saving in the $\geq 65$ years age group irrespective of the influenza definition used, highlighting its value in this risk group.

Data Availability Statement Data that are detailed in the Methods section or electronic supplementary material can be found in the list of references contained in the article. Enquiries regarding the model can be addressed to the corresponding author.

Acknowledgments The authors thank Dr Xu-Hao Zhang (GSK, Singapore) and Emily Lloyd (GSK, UK) for their contribution to the study, and Business and Decision Life Sciences platform for editorial assistance and publication coordination, on behalf of GSK. Nathalie Arts coordinated publication development and provided editorial support.

Author Contributions YKK, JYS, HJ, and LV designed the model. HK, LV, THK, and EH designed the computational framework. LV and HK analyzed the data. HK and LV carried out the implementation of the model and the calculations. YKK, JYS, LV, and EH wrote the article with input from all authors. THK and EH conceived the study and were in charge of the overall direction and planning.

\section{Compliance with Ethical Standards}

Funding GlaxoSmithKline Biologicals SA was the funding source, was involved in all stages of the study conduct and analysis, and funded all costs associated with the development and publishing of the present article (GSK study identifier: HO-15-16168). All authors had full access to the data and agreed with submission of the article for publication.

Conflicts of Interest LV is an employee of the GSK group of companies, and HJ was an employee of the GSK group of companies at the time this study was conducted. YKK, JYS, HK, and EH report grants from the GSK group of companies, while THK reports grant and personal fees from the GSK group of companies.

Open Access This article is distributed under the terms of the Creative Commons Attribution-NonCommercial 4.0 International License (http://creativecommons.org/licenses/by-nc/4.0/), which permits any noncommercial use, distribution, and reproduction in any medium, provided you give appropriate credit to the original author(s) and the source, provide a link to the Creative Commons license, and indicate if changes were made.

\section{References}

1. World Health Organization. Vaccines against influenza WHO position paper - November 2012. Wkly Epidemiol Rec. 2012;87(47):461-76.

2. Hay AJ, Gregory V, Douglas AR, Lin YP. The evolution of human influenza viruses. Philos Trans R Soc Lond B Biol Sci. 2001;356(1416):1861-70.

3. Ambrose CS, Levin MJ. The rationale for quadrivalent influenza vaccines. Hum Vaccin Immunother. 2012;8(1):81-8.

4. Su S, Chaves SS, Perez A, D'Mello T, Kirley PD, Yousey-Hindes $\mathrm{K}$, et al. Comparing clinical characteristics between hospitalized adults with laboratory-confirmed influenza $\mathrm{A}$ and $\mathrm{B}$ virus infection. Clin Infect Dis. 2014;59(2):252-5.

5. Chagvardieff A, Persico N, Marmillot C, Badiaga S, Charrel R, Roch A. Prospective comparative study of characteristics associated with influenza A and B in adults. Med Mal Infect. 2018;48(3):180-7.

6. Hong KW, Cheong HJ, Song JY, Noh JY, Yang TU, Kim WJ. Clinical manifestations of influenza A and B in children and adults at a tertiary hospital in Korea during the 2011-2012 season. Jpn J Infect Dis. 2015;68(1):20-6.

7. Tran D, Vaudry W, Moore D, Bettinger JA, Halperin SA, Scheifele DW, et al. Hospitalization for Influenza A Versus B. Pediatrics. 2016;138(3).

8. Tricco AC, Chit A, Soobiah C, Hallett D, Meier G, Chen MH, et al. Comparing influenza vaccine efficacy against mismatched and matched strains: a systematic review and meta-analysis. BMC Med. 2013;11:153.

9. Yun JW, Noh JY, Song JY, Chun C, Kim Y, Cheong HJ. The Korean Influenza National Immunization Program: history and Present Status. Infect Chemother. 2017;49(4):247-54

10. Korea Centers for Disease Control and Prevention (KCDC). $2017-$ 2018 Seasonal Influenza National Immunization Support Project Management Guidelines [in Korean]. 2017. Available at: http:// www.cdc.go.kr/CDC/together/CdcKrTogether0302.jsp?menuI ds=HOME001-MNU1154-MNU0725-MNU0088\&cid=75943. Accessed 28 Feb 2018.

11. Korea Centers for Disease Control and Prevention (KCDC). Elderly Immunization Program against Influenza in Korea, 2015. 2016. Available at: http://www.cdc.go.kr/CDC/info/CdcKrInfo0 301.jsp?menuIds=HOME001-MNU1154-MNU0005-MNU00 $37 \&$ cid $=67630$. Accessed 28 Feb 2018.

12. Korea Statistical Information Service - STATISTICS KOREA. Influenza vaccination rate for children and adolescents by sex and age (1-18 years) [in Korean]. 2018. Available at: http:// kosis.kr/statHtml/statHtml.do?orgId=117\&tblId=DT_11702 _N084\&conn_path=I2. Accessed 28 Feb 2018.

13. Van Bellinghen LA, Marijam A. Tannus Branco de Araujo G, Gomez J, Van Vlaenderen I. Cost-utility of quadrivalent versus trivalent influenza vaccine in Brazil: comparison of outcomes from different static model types. Braz J Infect Dis. 2018;22(1):1-10.

14. Vemer P, Corro Ramos I, van Voorn GA, Al MJ, Feenstra TL. AdViSHE: a Validation-Assessment Tool of Health-Economic Models for Decision Makers and Model Users. Pharmacoeconomics. 2016;34(4):349-61.

15. Bae EY, Lee EK. Pharmacoeconomic guidelines and their implementation in the positive list system in South Korea. Value Health. 2009; 12(Suppl 3):S36-41.

16. Bae S, Lee S, Bae EY, Jang S. Korean guidelines for pharmacoeconomic evaluation (second and updated version): consensus and compromise. Pharmacoeconomics. 2013;31(4):257-67. 
17. Health Insurance Review and Assessment Service. Guideline for economic evaluation for drugs [in Korean]. 2006. Available at: https://www.ispor.org/PEguidelines/source/Korean_PE_Guide lines_Korean_Version.pdf. Accessed 20 Jun 2018.

18. Korea Statistical Information Service - Statistics Korea. Projected Population by Age. 2017. Available at: http://kosis.kr/ statHtml/statHtml.do?orgId=101\&tblId=DT_1BPA001\&conn_ path $=I 2 \&$ language $=$ en. Accessed $28 \mathrm{Feb} 2018$.

19. Lee YH, Han K, Ko SH, Ko KS, Lee KU. Data Analytic Process of a Nationwide Population-Based Study Using National Health Information Database Established by National Health Insurance Service. Diabetes Metab J. 2016;40(1):79-82.

20. National Evidence-based Healthcare Collaborating Agency (NECA). Evaluation of the Korea seasonal influenza disease burden \& seasonal influenza vaccination effectiveness [in Korean]. 2009. Available at: http://www.chsc.or.kr/?post_type=refer ence \&p=5758. Accessed 5 Oct 2017.

21. Kim YK, Nyambat B, Hong YS, Lee CG, Lee JW, Kilgore PE. Burden of viral respiratory disease hospitalizations among children in a community of Seoul, Republic of Korea, 1995-2005. Scand J Infect Dis. 2008;40(11-12):946-53.

22. Im CS, Choi JH, Kim KM. Analysis on linking the sample cohort of the Korean National Health Insurance Service (NHIS) and data in Statistics Korea. Statistical Research Institute. [in Korean]. 2016. Available at: http://kostat.go.kr/edu/sri_kor_new/1/1/index .action?bmode $=$ download\&p_idx=396. Accessed 20 Jun 2018.

23. Korea Centers for Disease Control and Prevention (KCDC). Korean Influenza Surveillance Scheme - Weekly reports [in Korean]. 2017. Available at: http://cdc.go.kr/CDC/intro/CdcKr Intro 0201.jsp?menuIds=HOME001-MNU1154-MNU00 05-MNU0011\&cid=66382. Accessed 18 Apr 2017.

24. Jennings L, Huang QS, Barr I, Lee PI, Kim WJ, Buchy P, et al. Literature review of the epidemiology of influenza $B$ disease in 15 countries in the Asia-Pacific region. Influenza Other Respir Viruses. 2018;12(3):383-411.

25. Korea Statistical Information Service (KOSIS). Statistics Korea - GDP deflators. Available at: http://kosis.kr/statHtml/statHtml. do?orgId=101\&tblId=DT_2KAA602_OECD. Accessed 24 Jun 2018.

26. Ministry of Health and Welfare. Response to the article on flu vaccination for infants and young children [in Korean]. Available at: http://www.mohw.go.kr/m/noticeView.jsp?MENU_ $\mathrm{ID}=0404 \&$ cont_seq=335044\&page=1. Accessed $11 \mathrm{Jul} 2018$.

27. Choe YJ, Lee H, Lee HJ, Choi EH. Antiviral treatment of influenza in South Korea. Expert Rev Anti Infect Ther. 2015;13(6):741-9.

28. Song JY, Noh JY, Choi WS, Cheong HJ, Kim WJ. Antiviral therapy in seasonal influenza and 2009 H1N1 pandemic influenza: korean experiences and perspectives. Expert Rev Anti Infect Ther. 2015;13(11):1361-72.

29. Health Insurance Review and Assessment (HIRA) Service. Current drug payment list and salary cap. Available at: http://www. hira.or.kr/bbsDummy.do?pgmid=HIRAA030014050000\&brdSc $\mathrm{nBltNo}=4 \&$ brdBltNo$=1604 \&$ pageIndex $=1 \#$ none. Accessed 11 Jul 2018.

30. Choi WS, Lee J, Lee HY, Baek JH, Kim YK, Kee SK, et al. Clinical Practice Guideline for Antiviral Treatment and Chemoprophylaxis of Seasonal Influenza. Infect Chemother. 2012;44(4):233-49.

31. Demicheli V, Jefferson T, Al-Ansary LA, Ferroni E, Rivetti A, Di Pietrantonj C. Vaccines for preventing influenza in healthy adults. Cochrane Database Syst Rev. 2014;(3):CD001269.

32. Jefferson T, Di Pietrantonj C, Al-Ansary LA, Ferroni E, Thorning $S$, Thomas RE. Vaccines for preventing influenza in the elderly. Cochrane Database Syst Rev. 2010;(2):CD004876.

33. Jefferson T, Rivetti A, Di Pietrantonj C, Demicheli V, Ferroni E. Vaccines for preventing influenza in healthy children. Cochrane Database Syst Rev. 2012;(8):CD004879.
34. Claeys C, Zaman K, Dbaibo G, Li P, Izu A, Kosalaraksa P, et al. Prevention of vaccine-matched and mismatched influenza in children aged 6-35 months: a multinational randomised trial across five influenza seasons. The Lancet Child Adolescent Health. 2018;2(5):338-49.

35. Tisa V, Barberis I, Faccio V, Paganino C, Trucchi C, Martini M, et al. Quadrivalent influenza vaccine: a new opportunity to reduce the influenza burden. J Prev Med Hyg. 2016;57(1):E28-33.

36. Yang HJ, Cho SI. Influenza vaccination coverage among adults in Korea: 2008-2009 to 2011-2012 seasons. Int J Environ Res Public Health. 2014;11(12):12162-73.

37. Seo J, Lim J. Trends in influenza vaccination coverage rates in South Korea from 2005 to 2014: effect of public health policies on vaccination behavior. Vaccine. 2018;36(25):3666-73.

38. Mauskopf JA, Cates SC, Griffin AD, Neighbors DM, Lamb SC, Rutherford C. Cost effectiveness of zanamivir for the treatment of influenza in a high risk population in Australia. Pharmacoeconomics. 2000;17(6):611-20.

39. Thommes EW, Ismaila A, Chit A, Meier G, Bauch CT. Cost-effectiveness evaluation of quadrivalent influenza vaccines for seasonal influenza prevention: a dynamic modeling study of Canada and the United Kingdom. BMC Infect Dis. 2015;15:465.

40. Lee JY, Ock M, Kim SH, Go DS, Kim HJ, Jo MW. Healthadjusted life expectancy (HALE) in Korea: 2005-2011. J Korean Med Sci. 2016;31(Suppl 2):S139-49.

41. Neine M, Curran D. An Algorithm to develop correlated multivariate non-normal (E.G. Beta, Gamma, Log-Normal) distributions to be used in probabilistic sensitivity analyses (PSAS). Value Health. 2017;20(9):A757.

42. Korea Statistical Information Service-STATISTICS. Per capita GDP (current prices, won)-Main Annual Indicators (reference year 2010). 2017. Available at: http://kosis.kr/statHtml/statH tml.do?orgId=301\&tblId=DT_102Y002\&conn_path=I2\&langu age $=$ en. Accessed $28 \mathrm{Feb} 2018$.

43. Jamotte A, Clay E, Macabeo B, Caicedo A, Lopez JG, Bricks L, et al. Public health impact and economic benefits of quadrivalent influenza vaccine in Latin America. Hum Vaccin Immunother. 2017;13(4):877-88.

44. Cleemput I, Neyt M, Thiry N, De Laet C, Leys M. Using threshold values for cost per quality-adjusted life-year gained in healthcare decisions. Int J Technol Assess Health Care. 2011;27(1):71-6.

45. Shiroiwa T, Sung YK, Fukuda T, Lang HC, Bae SC, Tsutani K. International survey on willingness-to-pay (WTP) for one additional QALY gained: what is the threshold of cost effectiveness? Health Econ. 2010;19(4):422-37.

46. Grosse SD. Assessing cost-effectiveness in healthcare: history of the \$50,000 per QALY threshold. Expert Rev Pharmacoecon Outcomes Res. 2008;8(2):165-78.

47. Rawlins MD, Culyer AJ. National institute for clinical excellence and its value judgments. BMJ. 2004;329(7459):224-7.

48. Health Insurance Review \& Assessment (HIRA) Service. Assessment guideline for new drugs. July 2016 [in Korean]. Available at: https://www.hira.or.kr/bbs/bbsCDownLoad.do?apndN $\mathrm{o}=585 \&$ apndBrdBlt $\mathrm{No}=45767 \&$ apndB $\mathrm{rdTyNo}=1 \& \mathrm{apndB}$ $1 \mathrm{tNo}=83$. Accessed $24 \mathrm{Jul} 2018$.

49. Kim HS, Kang YJ, Ko SK, Kang HY. Decision making based on incremental cost-effectiveness ratio for anticancer drugs. J Health Tech Assess. 2014;2(1):68-73.

50. Gerlier L, Lamotte M, Greneche S, Lenne X, Carrat F, WeilOlivier C, et al. Assessment of public health and economic impact of intranasal live-attenuated influenza vaccination of children in France using a dynamic transmission model. Appl Health Econ Health Policy. 2017;15(2):261-76.

51. Baguelin M, Camacho A, Flasche S, Edmunds WJ. Extending the elderly- and risk-group programme of vaccination against seasonal 
influenza in England and Wales: a cost-effectiveness study. BMC Med. 2015;13:236.

52. Damm O, Eichner M, Rose MA, Knuf M, Wutzler P, Liese JG, et al. Public health impact and cost-effectiveness of intranasal live attenuated influenza vaccination of children in Germany. Eur J Health Econ. 2015;16(5):471-88.

53. de Boer PT, van Maanen BM, Damm O, Ultsch B, Dolk FCK, Crepey $\mathrm{P}$, et al. A systematic review of the health economic consequences of quadrivalent influenza vaccination. Expert Rev Pharmacoecon Outcomes Res. 2017;17(3):249-65.

54. Quinn E, Jit M, Newall AT. Key issues and challenges in estimating the impact and cost-effectiveness of quadrivalent influenza vaccination. Expert Rev Pharmacoecon Outcomes Res. 2014;14(3):425-35.

55. Chit A, Roiz J, Aballea S. An assessment of the expected costeffectiveness of quadrivalent influenza vaccines in Ontario, Canada Using a static model. PLoS One. 2015;10(7):e0133606.

56. Clements KM, Meier G, McGarry LJ, Pruttivarasin N, Misurski DA. Cost-effectiveness analysis of universal influenza vaccination with quadrivalent inactivated vaccine in the United States. Hum Vaccin Immunother. 2014;10(5):1171-80.
57. Meier G, Gregg M, Poulsen Nautrup B. Cost-effectiveness analysis of quadrivalent influenza vaccination in at-risk adults and the elderly: an updated analysis in the U.K. J Med Econ. 2015;18(9):746-61.

58. Thommes EW, Kruse M, Kohli M, Sharma R, Noorduyn SG. Review of seasonal influenza in Canada: burden of disease and the cost-effectiveness of quadrivalent inactivated influenza vaccines. Hum Vaccin Immunother. 2017;13(4):867-76.

59. Van Bellinghen LA, Meier G, Van Vlaenderen I. The potential cost-effectiveness of quadrivalent versus trivalent influenza vaccine in elderly people and clinical risk groups in the UK: a lifetime multi-cohort model. PLoS One. 2014;9(6):e98437.

60. Dolk C, Eichner M, Welte R, Anastassopoulou A, Van Bellinghen LA, Poulsen Nautrup B, et al. Cost-utility of quadrivalent versus trivalent influenza vaccine in Germany, using an individual-based dynamic transmission model. Pharmacoeconomics. 2016;34(12):1299-308.

61. Nagy L, Heikkinen T, Sackeyfio A, Pitman R. The clinical impact and cost effectiveness of quadrivalent versus trivalent influenza vaccination in finland. Pharmacoeconomics. 2016;34(9):939-51.

\section{Affiliations}

\section{Yun-Kyung Kim ${ }^{1}$. Joon Young Song ${ }^{2} \cdot$ Hyeongap Jang ${ }^{3} \cdot$ Tae Hyun Kim $^{4}\left(\mathbb{D} \cdot\right.$ Heejo Koo $^{5} \cdot$ Lijoy Varghese $^{6} \odot$. Euna $\operatorname{Han}^{5,7}$ (D)}

Euna Han

eunahan@yonsei.ac.kr; THKIM@yuhs.ac

1 Korea University Ansan Hospital, Ansan, Republic of Korea

2 Division of Infectious Diseases, Guro Hospital, Korea University College of Medicine, Seoul, Republic of Korea

3 GSK, Seoul, Republic of Korea

4 Graduate School of Public Health, Yonsei University, Seoul, Republic of Korea
5 College of Pharmacy, Yonsei Institute of Pharmaceutical Sciences, Yonsei University, Incheon, Republic of Korea

6 GSK, Singapore, Singapore

7 College of Pharmacy, Yonsei Institute of Pharmaceutical Sciences, Yonsei University, 162-1 Songdo-Dong, Yeonsu-Gu, Incheon, Republic of Korea 